\title{
New Stability Analysis for Linear Systems with Time-Varying Delay Based on Combined Convex Technique
}

\author{
Bin Yang and Chen-xin Fan \\ The School of Control Science and Engineering, Dalian University of Technology, Dalian 116023, China \\ Correspondence should be addressed to Chen-xin Fan; fanchenxin.ok@163.com
}

Received 8 March 2014; Accepted 5 August 2014

Academic Editor: Huaguang Zhang

Copyright ( 92015 B. Yang and C.-x. Fan. This is an open access article distributed under the Creative Commons Attribution License, which permits unrestricted use, distribution, and reproduction in any medium, provided the original work is properly cited.

A novel combined convex method is developed for the stability of linear systems with a time-varying delay. A new delay-dependent stability condition expressed in terms of linear matrix inequalities (LMIs) is derived by employing a dedicated constructed Lyapunov-Krasovskii functional (LKF), utilizing the Wirtinger inequality and the reciprocally convex approach to handle the integral term of quadratic quantities. Different from the previous convex techniques which only tackle the time-varying delay, our method adopts the idea of combined convex technique which can tackle not only the delay but also the delay variation. Four well-known examples are illustrated to show the effectiveness of the proposed results.

\section{Introduction}

In recent years, the stability of the time-delayed linear system is one of the hot issues in control theory, for time delay occurs in different physical, industrial, and engineering systems, such as aircraft, biological systems, population dynamics, and neural networks. It is well-known that time delay is often a source of the degradation of performance and/or the instability of the time-delayed linear system. Hence, the problem of the stability analysis of time-delayed systems has attracted considerable attention in recent years. For more details, see the literature [1-29].

Currently, many researchers have devoted time and effort to the stability analysis of linear-systems with time delay, and a great number of results on delay-dependent stability conditions for time-delayed systems have been reported in the briefs $[6,8,11,17,20,22,28,29]$ because it is well known that delay-dependent stability criteria which include the information on the size of time delay are generally less conservative than delay-independent ones when the size of time delay is small. The objective of the stability analysis is to find a less conservative condition to enlarge the feasibility region of stability criteria such that it guarantees asymptotic stability of time-delayed systems as large as possible. In order to reduce the conservatism of the stability criteria for linear time-delayed system, integral inequality lemma was used by Park and Ko [9]. He et al. presented some less conservative stability conditions using free-weighting matrix in $[11,23]$. Descriptor model transform method was presented by Fridman and Shaked in [12]; Jensen's inequality and delay decomposition method were used in $[17,20,21]$ and [30], respectively. Jensen's inequality introduces an undesirable conservatism in the stability conditions, so, some Wirtinger inequalities which allow consideration of more accurate integral inequalities are introduced by Seuret and Gouaisbaut to deal with the derivative of LKF recently in [18]. Notice that the reciprocal convex approach presented in [24] has been a popular method. Although this method can be more effective than earlier convex techniques in studying the timevarying delay systems, it still needs more improvements since it cannot tackle the delay variation or more complicated cases [15].

In the light of the discussion above, in this paper, the combined convex method which was presented in [31, 32] is further developed for the stability of the linear systems with time-varying delay. With the new method, both the time-varying delay and the variation of the delay can be tackled. We notice that some important terms are ignored during the construction of the LKF because of limitation of the previous method. First, we construct a new LKF and 
use reciprocal convex approach and Wirtinger inequality to handle the integral term of quadratic quantities, and then we derive the stability condition in terms of the sum of two firstorder convex functions with respect to the time-varying delay and its variation. Second, a novel delay-dependent stability criterion is presented in terms of LMIs which can be solved efficiently by convex optimization algorithm. Finally, four well-known examples are given to illustrate the effectiveness of the proposed method.

Throughout this paper, the following notations will be used: $C^{T}$ represents the transposition of matrix $C, \mathbb{R}^{n}$ denotes $n$-dimensional Euclidean space, and $\mathbb{R}^{n \times m}$ is the set of all $n \times m$ real matrices. $P>0$ means that $P$ is positively definite. Symbol $*$ represents the elements below the main diagonal of a symmetric block matrix. $\operatorname{Sym}(X)$ is defined as $\operatorname{Sym}(X)=$ $X+X^{T}$.

\section{Problem Statement}

Consider the following linear systems with time-varying delay:

$$
\begin{aligned}
& \dot{x}(t)=A x(t)+B x(t-h(t)), \quad t \geq 0, \\
& x(t)=\psi(t), \quad t \in[-h, 0],
\end{aligned}
$$

where $x(t) \in \mathbb{R}^{n}$ is the state vector, $A, B \in \mathbb{R}^{n \times n}$ are constant matrices with appropriate dimensions, $h(t)$ is the time-varying delay, and it is assumed to satisfy the following:

$$
\begin{aligned}
& \mathrm{C} 1: 0 \leq h(t) \leq h, \mu_{1} \leq \dot{h}(t) \leq \mu_{2}<1, \text { for all } t \geq 0 ; \\
& \mathrm{C} 2: 0 \leq h(t) \leq h \text {, for all } t \geq 0 .
\end{aligned}
$$

The initial condition $\psi(t)$ is a continuously differentiable function on $[-h, 0]$.

For $\mathrm{Cl}$, let us define $\nabla_{d}$ in the following set:

$$
\Psi_{d}:=\left\{\nabla_{d} \mid \nabla_{d} \in \operatorname{conv}\left\{\nabla_{d}^{1}, \nabla_{d}^{2}\right\}\right\}
$$

where conv denotes the convex hull, $\nabla_{d}^{1}=\mu_{1}$, and $\nabla_{d}^{2}=\mu_{2}$. Then, there exists a parameter $\theta>0$ such that $\dot{h}(t)$ can be expressed as convex combination of the vertices as follows:

$$
\dot{h}(t)=\theta \nabla_{d}^{1}+(1-\theta) \nabla_{d}^{2}
$$

If a matrix $M_{|\dot{h}(t)|}$ is affinely dependent on $\dot{h}(t)$, then $M_{|\dot{h}(t)|}$ can be expressed as convex combinations of the vertices

$$
M_{[\dot{h}(t)]}=\theta M_{\left[\nabla_{d}^{1}\right]}+(1-\theta) M_{\left[\nabla_{d}^{2}\right]} \cdot
$$

From (4), if a stability condition is affinely dependent on $\dot{h}(t)$, then it needs only to check the vertex values of $\dot{h}(t)$ instead of checking all values of $\dot{h}(t)$ [33].
Before deriving the main results, the following lemmas are stated, which will be used in the proof of the main results.

Lemma 1 (see [18]). For a given matrix $R>0$, the following inequality holds for all continuously differentiable functions $\omega$ in $[a, b] \rightarrow \mathbb{R}^{n}:$

$$
\begin{aligned}
\int_{a}^{b} \dot{\omega}^{T}(u) R \dot{\omega}(u) d u \geq & \frac{1}{b-a}(\omega(b)-\omega(a))^{T} R(\omega(b)-\omega(a)) \\
& +\frac{3}{b-a} \Psi^{T} R \Psi
\end{aligned}
$$

where $\Psi=\omega(b)+\omega(a)-(2 /(b-a)) \int_{a}^{b} \omega(u) d u$.

Lemma 2 (see [22]). Suppose that $\Omega, \Xi_{1 i}, \Xi_{2 i}(i=1,2)$ are the constant matrices of appropriate dimensions, $\alpha \in[0,1]$, and $\beta \in[0,1]$, then $\Omega+\left[\alpha \Xi_{11}+(1-\alpha) \Xi_{12}\right]+\left[\beta \Xi_{21}+(1-\beta) \Xi_{22}\right]<0$ holds, if the following four inequalities hold simultaneously:

$$
\begin{array}{ll}
\Omega+\Xi_{11}+\Xi_{21}<0, & \Omega+\Xi_{11}+\Xi_{22}<0, \\
\Omega+\Xi_{12}+\Xi_{21}<0, & \Omega+\Xi_{12}+\Xi_{22}<0 .
\end{array}
$$

The reciprocally convex combination inequality provided in Park et al. [24] is used in this paper. This inequality has been reformulated by Seuret and Gouaisbaut [18] and is stated in Lemma 3.

Lemma 3. For given positive integers $n, m, a$ scalar $\delta$ in the interval $(0,1)$, a given $n \times n$ matrix $R>0$, and two matrices $W_{1}$ and $W_{2}$ in $\mathbb{R}^{n \times m}$. Define, for all vectors $\xi$ in $\mathbb{R}^{m}$, the function $\Theta(\alpha, R)$ given by the following:

$$
\Theta(\delta, R)=\frac{1}{\delta} \xi^{T} W_{1}^{T} R W_{1} \xi+\frac{1}{1-\delta} \xi^{T} W_{2}^{T} R W_{2} \xi .
$$

Then, if there exists a matrix $X$ in $\mathbb{R}^{n \times n}$ such that $\left[\begin{array}{ll}R & X \\ * & R\end{array}\right]>0$, then the following inequality holds:

$$
\min _{\delta \in(0,1)} \Theta(\delta, R) \geq\left[\begin{array}{l}
W_{1} \xi \\
W_{2} \xi
\end{array}\right]^{T}\left[\begin{array}{ll}
R & X \\
* & R
\end{array}\right]\left[\begin{array}{l}
W_{1} \xi \\
W_{2} \xi
\end{array}\right] .
$$

\section{Main Result}

The main objective of this section is to achieve a less conservative condition such that it can guarantee the stability of system (1) under the constraint C1. First, we estimate the derivative of Lyapunov functional less conservatively by constructing a new augmented LKF; then, with the Wirtinger inequality and the newly developed combined convex technique, the improved stability results are derived, which are less conservative than some existing ones.

For simplicity of matrix representation, we set block entry matrices $e_{i}(i=1, \ldots, 7) \in \mathbb{R}^{7 n \times n}$ (e.g., $e_{2}^{T}=$ $\left[\begin{array}{lllllll}0 & I & 0 & 0 & 0 & 0 & 0\end{array}\right]$ ) and we define the following: 


$$
\begin{aligned}
& \eta^{T}(t)=\left[\begin{array}{lll}
x^{T}(t) & x^{T}(t-h) & \int_{t-h}^{t} x^{T}(s) d s
\end{array}\right], \quad \phi^{T}(t, s)=\left[\begin{array}{lll}
x^{T}(t) & x^{T}(s) & \dot{x}^{T}(s)
\end{array}\right], \quad \varphi^{T}(t)=\left[\begin{array}{lll}
\dot{x}^{T}(t) & 0 & 0
\end{array}\right],
\end{aligned}
$$

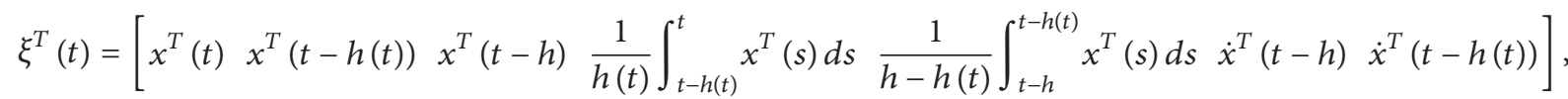

and construct the following LKF:

$$
V\left(x_{t}\right)=\sum_{i=1}^{4} V_{i}\left(x_{t}\right)
$$

where

$$
\begin{aligned}
V_{1}\left(x_{t}\right)= & \eta^{T}(t) P \eta(t), \\
V_{2}\left(x_{t}\right)= & \int_{t-h(t)}^{t}\left[\begin{array}{c}
\phi(t, s) \\
x(t-h)
\end{array}\right]^{T} Q\left[\begin{array}{c}
\phi(t, s) \\
x(t-h)
\end{array}\right] d s \\
& +\int_{t-h}^{t-h(t)}\left[\begin{array}{c}
\phi(t, s) \\
x(t-h)
\end{array}\right]^{T} M\left[\begin{array}{c}
\phi(t, s) \\
x(t-h)
\end{array}\right] d s, \\
V_{3}\left(x_{t}\right)= & \int_{t-h}^{t} \phi(t, s)^{T} Z \phi(t, s) d s, \\
V_{4}\left(x_{t}\right)= & h \int_{t-h}^{t} \int_{s}^{t} \dot{x}^{T}(u) R \dot{x}(u) d u d s,
\end{aligned}
$$

and here $P \in \mathbb{R}^{3 n \times 3 n}, Q \in \mathbb{R}^{4 n \times 4 n}, M \in \mathbb{R}^{4 n \times 4 n}, Z \in \mathbb{R}^{3 n \times 3 n}$, and $R \in \mathbb{R}^{n \times n}$.

Theorem 4. For given scalar $h \geq 0, \mu_{1}$ and $\mu_{2}$ with $C 1$, the system (1) is asymptotically stable, if there exist symmetric positive definite matrices $P \in \mathbb{R}^{3 n \times 3 n}, Q \in \mathbb{R}^{4 n \times 4 n}, M \in \mathbb{R}^{4 n \times 4 n}$, $Z \in \mathbb{R}^{3 n \times 3 n}$, and $R \in \mathbb{R}^{n \times n}$ and any matrices $S_{i j} \in \mathbb{R}^{n \times n}(i, j=$ $1,2)$, such that the following LMIs are feasible:

$$
\begin{gathered}
\Sigma_{\left[\nabla_{d}^{k}\right]}+h \Sigma_{1}+\Xi<0, \quad \forall k=1,2, \\
\Sigma_{\left[\nabla_{d}^{k}\right]}+h \Sigma_{2}+\Xi<0, \quad \forall k=1,2, \\
\Phi>0,
\end{gathered}
$$

where

$$
\begin{aligned}
& A_{C}^{T}=\left[\begin{array}{lllllll}
A & B & 0 & 0 & 0 & 0 & 0
\end{array}\right]^{T}, \\
& \Gamma=\left[\begin{array}{llll}
e_{1}-e_{2} & e_{1}+e_{2}-2 e_{4} & e_{2}-e_{3} & e_{2}+e_{3}-2 e_{5}
\end{array}\right]^{T},
\end{aligned}
$$

$$
\begin{aligned}
& \Sigma_{\left[\nabla_{d}^{k}\right]}=-\left(1-\nabla_{d}^{k}\right)\left[\begin{array}{llll}
e_{1} & e_{2} & e_{7} & e_{3}
\end{array}\right] Q\left[\begin{array}{llll}
e_{1} & e_{2} & e_{7} & e_{3}
\end{array}\right]^{T} \\
& +\left(1-\nabla_{d}^{k}\right)\left[\begin{array}{llll}
e_{1} & e_{2} & e_{7} & e_{3}
\end{array}\right] M\left[\begin{array}{llll}
e_{1} & e_{2} & e_{7} & e_{3}
\end{array}\right]^{T}, \\
& \Sigma_{1}=\operatorname{Sym}\left(\left[\begin{array}{lll}
0 & 0 & e_{4}
\end{array}\right] P\left[\begin{array}{lll}
A_{C}^{T} & e_{6} & e_{1}-e_{3}
\end{array}\right]^{T}\right) \\
& +\operatorname{Sym}\left(\left[\begin{array}{llll}
e_{1} & e_{4} & 0 & e_{3}
\end{array}\right] Q\left[\begin{array}{llll}
A_{C}^{T} & 0 & 0 & e_{6}
\end{array}\right]^{T}\right) \\
& +\operatorname{Sym}\left(\left[\begin{array}{lll}
0 & e_{4} & 0
\end{array}\right] Z\left[\begin{array}{lll}
A_{C}^{T} & 0 & 0
\end{array}\right]^{T}\right), \\
& \Sigma_{2}=\operatorname{Sym}\left(\left[\begin{array}{lll}
0 & 0 & e_{5}
\end{array}\right] P\left[\begin{array}{lll}
A_{C}^{T} & e_{6} & e_{1}
\end{array}-e_{3}\right]^{T}\right) \\
& +\operatorname{Sym}\left(\left[\begin{array}{llll}
e_{1} & e_{5} & 0 & e_{3}
\end{array}\right] M\left[\begin{array}{llll}
A_{C}^{T} & 0 & 0 & e_{6}
\end{array}\right]^{T}\right) \\
& +\operatorname{Sym}\left(\left[\begin{array}{lll}
0 & e_{5} & 0
\end{array}\right] Z\left[\begin{array}{lll}
A_{C}^{T} & 0 & 0
\end{array}\right]^{T}\right), \\
& \Xi=\operatorname{Sym}\left(\left[\begin{array}{lll}
e_{1} & e_{3} & 0
\end{array}\right] P\left[\begin{array}{lll}
A_{C}^{T} & e_{6} & e_{1}-e_{3}
\end{array}\right]^{T}\right) \\
& +\left[\begin{array}{llll}
e_{1} & e_{1} & A_{C}^{T} & e_{3}
\end{array}\right] Q\left[\begin{array}{llll}
e_{1} & e_{1} & A_{C}^{T} & e_{3}
\end{array}\right]^{T} \\
& +\operatorname{Sym}\left(\left[\begin{array}{llll}
0 & 0 & e_{1}-e_{2} & 0
\end{array}\right] Q\left[\begin{array}{llll}
A_{C}^{T} & 0 & 0 & e_{6}
\end{array}\right]^{T}\right) \\
& -\left[\begin{array}{llll}
e_{1} & e_{3} & e_{6} & e_{3}
\end{array}\right] M\left[\begin{array}{llll}
e_{1} & e_{3} & e_{6} & e_{3}
\end{array}\right]^{T} \\
& +\operatorname{Sym}\left(\left[\begin{array}{llll}
0 & 0 & e_{2}-e_{3} & 0
\end{array}\right] M\left[\begin{array}{llll}
A_{C}^{T} & 0 & 0 & e_{6}
\end{array}\right]^{T}\right) \\
& +\left[\begin{array}{lll}
e_{1} & e_{1} & A_{C}^{T}
\end{array}\right] Z\left[\begin{array}{lll}
e_{1} & e_{1} & A_{C}^{T}
\end{array}\right]^{T} \\
& -\left[\begin{array}{lll}
e_{1} & e_{3} & e_{6}
\end{array}\right] Z\left[\begin{array}{lll}
e_{1} & e_{3} & e_{6}
\end{array}\right]^{T}
\end{aligned}
$$

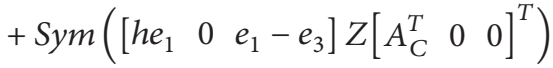

$$
\begin{aligned}
& +h^{2} A_{C}^{T} R A_{C}-\Gamma^{T} \Phi \Gamma \text {, }
\end{aligned}
$$$$
\Phi=\left[\begin{array}{cc}
\Theta & S \\
* & \Theta
\end{array}\right], \quad \Theta=\left[\begin{array}{cc}
R & 0 \\
0 & 3 R
\end{array}\right], S=\left[\begin{array}{ll}
S_{11} & S_{12} \\
S_{21} & S_{22}
\end{array}\right] .
$$

Proof. Taking the derivative of $V\left(x_{t}\right)$ with respect to $t$ along the solutions of system (1) yields 


$$
\begin{aligned}
& \dot{V}_{1}\left(x_{t}\right)=2 \eta^{T}(t) P\left[\begin{array}{c}
\dot{x}(t) \\
\dot{x}(t-h) \\
x(t)-x(t-h)
\end{array}\right] \\
& =\xi^{T}(t)\left\{\operatorname{Sym}\left(\left[\begin{array}{lll}
e_{1} & e_{3} & h(t) e_{4}+(h-h(t)) e_{5}
\end{array}\right] P\left[\begin{array}{lll}
A_{C}^{T} & e_{6} & e_{1}-e_{3}
\end{array}\right]^{T}\right)\right\} \xi(t), \\
& \dot{V}_{2}\left(x_{t}\right)=\left[\begin{array}{r}
\phi(t, t) \\
x(t-h)
\end{array}\right]^{T} Q\left[\begin{array}{r}
\phi(t, t) \\
x(t-h)
\end{array}\right]-(1-\dot{h}(t))\left[\begin{array}{c}
\phi(t, t-h(t)) \\
x(t-h)
\end{array}\right]^{T} Q\left[\begin{array}{c}
\phi(t, t-h(t)) \\
x(t-h)
\end{array}\right] \\
& +2 \int_{t-h(t)}^{t}\left[\begin{array}{c}
\phi(t, s) \\
x(t-h)
\end{array}\right]^{T} Q\left[\begin{array}{c}
\varphi(t) \\
\dot{x}(t-h)
\end{array}\right] d s+(1-\dot{h}(t))\left[\begin{array}{c}
\phi(t, t-h(t)) \\
x(t-h)
\end{array}\right]^{T} M\left[\begin{array}{c}
\phi(t, t-h(t)) \\
x(t-h)
\end{array}\right] \\
& -\left[\begin{array}{c}
\phi(t, t-h) \\
x(t-h)
\end{array}\right]^{T} M\left[\begin{array}{c}
\phi(t, t-h) \\
x(t-h)
\end{array}\right]+2 \int_{t-h}^{t-h(t)}\left[\begin{array}{c}
\phi(t, s) \\
x(t-h)
\end{array}\right]^{T} M\left[\begin{array}{c}
\varphi(t) \\
\dot{x}(t-h)
\end{array}\right] d s \\
& =\xi^{T}(t)\left\{\left[\begin{array}{llll}
e_{1} & e_{1} & A_{C}^{T} & e_{3}
\end{array}\right] Q\left[\begin{array}{llll}
e_{1} & e_{1} & A_{C}^{T} & e_{3}
\end{array}\right]^{T}-(1-\dot{h}(t))\left[\begin{array}{llll}
e_{1} & e_{2} & e_{7} & e_{3}
\end{array}\right] Q\left[\begin{array}{llll}
e_{1} & e_{2} & e_{7} & e_{3}
\end{array}\right]^{T}\right. \\
& +\operatorname{Sym}\left(\left[\begin{array}{llll}
h(t) e_{1} & h(t) e_{4} & e_{1}-e_{2} & h(t) e_{3}
\end{array}\right] Q\left[\begin{array}{llll}
A_{C}^{T} & 0 & 0 & e_{6}
\end{array}\right]^{T}\right) \\
& +(1-\dot{h}(t))\left[\begin{array}{llll}
e_{1} & e_{2} & e_{7} & e_{3}
\end{array}\right] \times M\left[\begin{array}{llll}
e_{1} & e_{2} & e_{7} & e_{3}
\end{array}\right]^{T}-\left[\begin{array}{llll}
e_{1} & e_{3} & e_{6} & e_{3}
\end{array}\right] M\left[\begin{array}{llll}
e_{1} & e_{3} & e_{6} & e_{3}
\end{array}\right]^{T} \\
& \left.+\operatorname{Sym}\left(\left[(h-h(t)) e_{1}(h-h(t)) e_{5} e_{2}-e_{3}(h-h(t)) e_{3}\right] M\left[\begin{array}{llll}
A_{C}^{T} & 0 & 0 & e_{6}
\end{array}\right]^{T}\right)\right\} \xi(t), \\
& \dot{V}_{3}\left(x_{t}\right)=\phi(t, t)^{T} Z \phi(t, t)-\phi(t, t-h)^{T} Z \phi(t, t-h)+2 \int_{t-h}^{t} \phi(t, s)^{T} Z \varphi(t) d s \\
& =\xi^{T}(t)\left\{\left[\begin{array}{lll}
e_{1} & e_{1} & A_{C}^{T}
\end{array}\right] Z\left[\begin{array}{lll}
e_{1} & e_{1} & A_{C}^{T}
\end{array}\right]^{T}-\left[\begin{array}{lll}
e_{1} & e_{3} & e_{6}
\end{array}\right] Z\left[\begin{array}{lll}
e_{1} & e_{3} & e_{6}
\end{array}\right]^{T}\right. \\
& \left.+\operatorname{Sym}\left(\left[\begin{array}{lll}
h e_{1} & h(t) e_{4}+(h-h(t)) e_{5} & e_{1}-e_{3}
\end{array}\right] Z\left[\begin{array}{lll}
A_{C}^{T} & 0 & 0
\end{array}\right]^{T}\right)\right\} \xi(t) .
\end{aligned}
$$

Finally, $\dot{V}_{4}$ is easily obtained as

$$
\dot{V}_{4}\left(x_{t}\right)=h^{2} \dot{x}^{T}(t) R \dot{x}(t)-h \int_{t-h}^{t} \dot{x}^{T}(s) R \dot{x}(s) d s .
$$

Using Lemmas 1 and 3 yields

$$
\begin{aligned}
& -h \int_{t-h}^{t} \dot{x}^{T}(s) R \dot{x}(s) d s \\
& =-h \int_{t-h(t)}^{t} \dot{x}^{T}(s) R \dot{x}(s) d s-h \int_{t-h}^{t-h(t)} \dot{x}^{T}(s) R \dot{x}(s) d s \\
& \leq-\frac{h}{h(t)}[x(t)-x(t-h(t))]^{T} R[x(t)-x(t-h(t))] \\
& -\frac{h}{h-h(t)}[x(t-h(t))-x(t-h)]^{T}
\end{aligned}
$$

$$
\begin{aligned}
& \times R[x(t-h(t))-x(t-h)] \\
& -\frac{3 h}{h(t)}\left[x(t)+x(t-h(t))-\frac{2}{h(t)} \int_{t-h(t)}^{t} x(s) d s\right]^{T} \\
& \times R\left[x(t)+x(t-h(t))-\frac{2}{h(t)} \int_{t-h(t)}^{t} x(s) d s\right] \\
& -\frac{3 h}{h-h(t)} \\
& \times\left[x(t-h(t))+x(t-h)-\frac{2}{h-h(t)} \int_{t-h}^{t-h(t)} x(s) d s\right]^{T} \\
& \times R\left[x(t-h(t))+x(t-h)-\frac{2}{h-h(t)} \int_{t-h}^{t-h(t)} x(s) d s\right]
\end{aligned}
$$




$$
\begin{aligned}
& =-\frac{h}{h(t)}\left[\begin{array}{c}
x(t)-x(t-h(t)) \\
x(t)+x(t-h(t))-\frac{2}{h(t)} \int_{t-h(t)}^{t} x(s) d s
\end{array}\right]^{T} \\
& \times \Theta\left[\begin{array}{c}
x(t)-x(t-h(t)) \\
x(t)+x(t-h(t))-\frac{2}{h(t)} \int_{t-h(t)}^{t} x(s) d s
\end{array}\right] \\
& -\frac{h}{h-h(t)} \\
& \times\left[\begin{array}{c}
x(t-h(t))-x(t-h) \\
x(t-h(t))+x(t-h)-\frac{2}{h-h(t)} \int_{t-h}^{t-h(t)} x(s) d s
\end{array}\right]^{T} \\
& \times \Theta\left[\begin{array}{c}
x(t-h(t))-x(t-h) \\
x(t-h(t))+x(t-h)-\frac{2}{h-h(t)} \int_{t-h}^{t-h(t)} x(s) d s
\end{array}\right] \\
& \leq-\left[\begin{array}{c}
x(t)-x(t-h(t)) \\
x(t)+x(t-h(t))-\frac{2}{h(t)} \int_{t-h(t)}^{t} x(s) d s \\
x(t-h(t))-x(t-h) \\
x(t-h(t))+x(t-h)-\frac{2}{h-h(t)} \int_{t-h}^{t-h(t)} x(s) d s
\end{array}\right]^{T} \\
& \times \Phi\left[\begin{array}{c}
x(t)-x 3(t-h(t)) \\
x(t)+x(t-h(t))-\frac{2}{h(t)} \int_{t-h(t)}^{t} x(s) d s \\
x(t-h(t))-x(t-h) \\
x(t-h(t))+x(t-h)-\frac{2}{h-h(t)} \int_{t-h}^{t-h(t)} x(s) d s
\end{array}\right], \\
& \dot{V}_{4}\left(x_{t}\right) \\
& \leq \xi^{T}(t) \\
& \times\left\{h^{2} A_{C}^{T} R A_{C^{-}}\left[\begin{array}{c}
e_{1}-e_{2} \\
e_{1}+e_{2}-2 e_{4} \\
e_{2}-e_{3} \\
e_{2}+e_{3}-2 e_{5}
\end{array}\right]^{T} \Phi\left[\begin{array}{c}
e_{1}-e_{2} \\
e_{1}+e_{2}-2 e_{4} \\
e_{2}-e_{3} \\
e_{2}+e_{3}-2 e_{5}
\end{array}\right]\right\} \xi(t) \\
& =\xi^{T}(t)\left\{h^{2} A_{C}^{T} R A_{C}-\Gamma^{T} \Phi \Gamma\right\} \xi(t) .
\end{aligned}
$$

Now, combining with (16)-(18), we get

$$
\dot{V}\left(x_{t}\right) \leq \xi^{T}(t)\left(\Sigma_{[\dot{h}(t)]}+\beta h \Sigma_{1}+(1-\beta) h \Sigma_{2}+\Xi\right) \xi(t),
$$

where

$$
\begin{gathered}
\Sigma_{[\dot{h}(t)]}=-(1-\dot{h}(t))\left[\begin{array}{llll}
e_{1} & e_{2} & e_{7} & e_{3}
\end{array}\right] Q\left[\begin{array}{llll}
e_{1} & e_{2} & e_{7} & e_{3}
\end{array}\right]^{T} \\
+(1-\dot{h}(t))\left[\begin{array}{llll}
e_{1} & e_{2} & e_{7} & e_{3}
\end{array}\right] M\left[\begin{array}{llll}
e_{1} & e_{2} & e_{7} & e_{3}
\end{array}\right]^{T} \\
\beta=\frac{h(t)}{h} .
\end{gathered}
$$

It is clear that $\Sigma_{[\dot{h}(t)]}+\beta h \Sigma_{1}+(1-\beta) h \Sigma_{2}+\Xi<0$ implies $\dot{V}\left(x_{t}\right)<0$, which means that system (1) is asymptotically stable. Notice that $\Sigma_{[\dot{h}(t)]}$ is affine and a convex combination on $\dot{h}(t)$ satisfying $\mu_{1} \leq \dot{h}(t) \leq \mu_{2}$, then $\Sigma_{[\dot{h}(t)]}$ can be expressed as convex combinations of the vertices $\Sigma_{[h(t)]}=$ $\alpha \Sigma_{\left[\nabla_{d}^{1}\right]}+(1-\alpha) \Sigma_{\left[\nabla_{d}^{2}\right]}, \alpha \in[0,1]$. Utilizing Lemma 2 , then $\Sigma_{[\dot{h}(t)]}+\beta h \Sigma_{1}+(1-\beta) h \Sigma_{2}+\Xi<0$ holds, if and only if (12) and (13) hold. This completes our proof.

Remark 5. Recently, the reciprocally convex optimization technique and Wirtinger inequality to reduce the conservatism of stability criteria for linear systems with timevarying delay were proposed in $[24,25,28]$ and $[18,21]$, respectively, and these methods were utilized in (18). In Lemma 1, it can be noticed that the term $(1 /(b-a))(\omega(b)-$ $\omega(a))^{T} R(\omega(b)-\omega(a))$ is equal to Jensen's inequality and that the newly appeared term $(3 /(b-a)) \Psi^{T} R \Psi$ can reduce the LKF enlargement of the estimation. The usage of reciprocally convex optimization method avoids the enlargement of $h(t)$ and $h-h(t)$ while only introducing matrix $S$. Then, the convex optimization method is used to handle $\dot{V}\left(x_{t}\right)$. During the proof procedure above, the dedicated constructed LKF (11) has full information on the systems.

Remark 6. Furthermore, we introduce terms $x(t), x(t-h)$ in $V_{2}$. Therefore, more information on the cross terms in $x(t), \dot{x}(t)$ and $x(t-h), \dot{x}(t-h)$ is utilized. To reduce the conservatism, the term $\int_{t-h}^{t-h(t)}\left[\begin{array}{c}\phi(t, s) \\ x(t-h)\end{array}\right]^{T} M\left[\begin{array}{c}\phi(t, s) \\ x(t-h)\end{array}\right] d s$ is chosen as LKF when $\mu_{1} \leq \dot{h}(t) \leq \mu_{2}$. These considerations highlight the main differences in the construction of the LKF candidate in this paper.

Remark 7. When $h(t)$ is not differentiable and $\dot{h}(t)$ is unknown, the state $\dot{x}(t-h(t))$ cannot be utilized as the augmented vector $\xi(t)$ by the methods presented in the proof of Theorem 4 . Thus, we should modify the LKF which includes the term $\dot{x}(t-h(t))$, so we set $Q, M=0$. Therefore, the corresponding stability criterion for $\mathrm{C} 2$ will be introduced as Corollary 8.

In Corollary 8, block entry matrices $\widetilde{e}_{i}(t) \in \mathbb{R}^{6 n \times n}$ will be used and the following notations are defined for the sake of simplicity of matrix notation: 
TABLE 1: Delay bounds $h$ with different $\mu_{2}$ and $\mu_{1}=-\mu_{2}$.

\begin{tabular}{lcccccc}
\hline$\mu_{2}$ & 0.0 & 0.1 & 0.2 & 0.5 & 0.8 & $\mu_{2} \geq 1$ or unknown \\
\hline Fridman and Shaked [12] & 4.472 & 3.604 & 3.303 & 2.008 & 1.364 & 0.999 \\
He et al. [11] & 4.472 & 3.605 & 3.039 & 2.043 & 1.492 & 1.345 \\
Sun et al. (2010) [20] & 4.472 & 3.611 & 3.047 & 2.072 & 1.590 & 1.529 \\
Seuret and Gouaisbaut [18, Theorem 7] & 6.059 & 4.703 & 3.834 & 2.420 & 2.137 & 2.128 \\
Theorem 4 & 6.059 & 4.733 & 3.932 & 2.891 & 2.592 & - \\
Corollary 8 & - & - & - & - & - & 2.113 \\
\hline
\end{tabular}

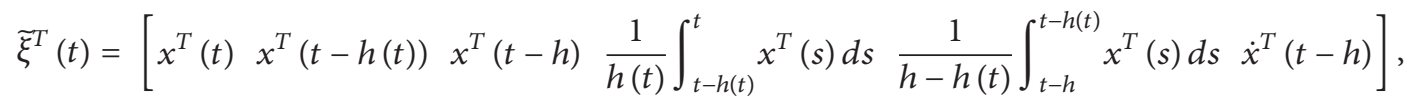

$$
\begin{aligned}
& \widetilde{\Sigma}_{1}=\operatorname{Sym}\left(\left[\begin{array}{lll}
0 & 0 & \widetilde{e}_{4}
\end{array}\right] P\left[\begin{array}{lll}
\widetilde{A}_{C}^{T} & \widetilde{e}_{6} & \widetilde{e}_{1}-\widetilde{e}_{3}
\end{array}\right]^{T}\right)+\operatorname{Sym}\left(\left[\begin{array}{lll}
0 & \widetilde{e}_{4} & 0
\end{array}\right] Z\left[\begin{array}{lll}
\widetilde{A}_{C}^{T} & 0 & 0
\end{array}\right]^{T}\right),
\end{aligned}
$$

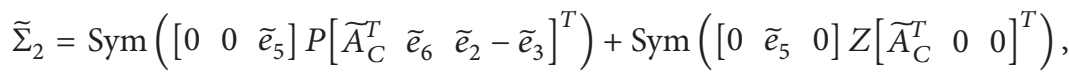

$$
\begin{aligned}
& \widetilde{A}_{C}^{T}=\left[\begin{array}{llllll}
A & B & 0 & 0 & 0 & 0
\end{array}\right]^{T} \text {, } \\
& \widetilde{\Xi}=\operatorname{Sym}\left(\left[\begin{array}{lll}
\widetilde{e}_{1} & \widetilde{e}_{3} & 0
\end{array}\right] P\left[\begin{array}{lll}
\widetilde{A}_{C}^{T} & \widetilde{e}_{6} & \widetilde{e}_{1}-\widetilde{e}_{3}
\end{array}\right]^{T}\right)+\left[\begin{array}{lll}
\widetilde{e}_{1} & \widetilde{e}_{1} & \widetilde{A}_{C}^{T}
\end{array}\right] Z\left[\begin{array}{lll}
\widetilde{e}_{1} & \widetilde{e}_{1} & \widetilde{A}_{C}^{T}
\end{array}\right]^{T}-\left[\begin{array}{lll}
\widetilde{e}_{1} & \widetilde{e}_{3} & \widetilde{e}_{6}
\end{array}\right] Z\left[\begin{array}{lll}
\widetilde{e}_{1} & \widetilde{e}_{3} & \widetilde{e}_{6}
\end{array}\right]^{T} \\
& +\operatorname{Sym}\left(\left[\begin{array}{lll}
h \widetilde{e}_{1} & 0 & \widetilde{e}_{1}-\widetilde{e}_{3}
\end{array}\right] Z\left[\begin{array}{lll}
\widetilde{A}_{C}^{T} & 0 & 0
\end{array}\right]^{T}\right)+h^{2} A_{C}^{T} R A_{C}-\Gamma^{T} \Phi \Gamma .
\end{aligned}
$$

Corollary 8. For given scalar $h \geq 0$ with C2, the system (1) is asymptotically stable, if there exist symmetric positive definite matrices $P \in \mathbb{R}^{3 n \times 3 n}, Z \in \mathbb{R}^{3 n \times 3 n}$, and $R \in \mathbb{R}^{n \times n}$. and any matrices $S_{i j} \in \mathbb{R}^{n \times n}(i, j=1,2)$, such that the following LMIs are feasible:

$$
\begin{gathered}
\widetilde{\Sigma}_{1}+\widetilde{\Xi}<0, \\
\widetilde{\Sigma}_{2}+\widetilde{\Xi}<0, \\
\Phi>0,
\end{gathered}
$$

where $\widetilde{\Sigma}_{1}, \widetilde{\Sigma}_{2}$, $\widetilde{\Xi}$ are defined in (21) and other matrices are defined in Theorem 4.

Proof.

$$
\widetilde{V}\left(x_{t}\right)=\sum_{i=1}^{3} \widetilde{V}_{i}\left(x_{t}\right)
$$

where

$$
\begin{gathered}
\widetilde{V}_{1}\left(x_{t}\right)=\eta^{T}(t) P \eta(t), \\
\widetilde{V}_{2}\left(x_{t}\right)=\int_{t-h}^{t} \phi(t, s)^{T} Z \phi(t, s) d s, \\
\widetilde{V}_{3}\left(x_{t}\right)=h \int_{t-h}^{t} \int_{s}^{t} \dot{x}^{T}(u) R \dot{x}(u) d u d s .
\end{gathered}
$$

Finally, we can get

$$
\dot{\widetilde{V}}\left(x_{t}\right) \leq \widetilde{\xi}^{T}(t)\left(\beta h \widetilde{\Sigma}_{1}+(1-\beta) h \widetilde{\Sigma}_{2}+\widetilde{\Xi}\right) \widetilde{\xi}(t)
$$

with the augmented vector $\widetilde{\xi}^{T}(t)$ defined in (21) and it is easy to see that $\beta h \widetilde{\Sigma}_{1}+(1-\beta) h \widetilde{\Sigma}_{2}+\widetilde{\Xi}$ is a convex combination, so we can see that (22) can guarantee the asymptotic stability for system (1).

\section{Numerical Examples}

In this section, four examples are given to show the effectiveness of the proposed method.

Example 9. Consider the linear system (1) with the parameters

$$
A=\left[\begin{array}{cc}
-2 & 0 \\
0 & -0.9
\end{array}\right], \quad B=\left[\begin{array}{cc}
-1 & 0 \\
-1 & -1
\end{array}\right] .
$$

This system is a well-known delay-dependent stable system which has the analytical maximum allowable delay bound $h_{\max }=6.1721$ when $\dot{h}(t)=0, \forall t \geq 0$. With the conditions $0 \leq h(t) \leq h$ and $\mu_{1} \leq \dot{h}(t) \leq \mu_{2}<1$, Table 1 shows that our results obtained by Theorem 4 improve the allowable maximum size of the delay for various $\mu_{2}$. For the case $\mu_{2}$ is unknown, they show less conservatism compared to the results of $[11,12,20]$; they also show that the combined convex technique and the Wirtinger inequality methods are effective but fall short compared to the results of [18] for this case. 
TABLE 2: Delay bounds $h$ with different $\mu_{2}$ and $\mu_{1}=0$.

\begin{tabular}{lccccc}
\hline$\mu_{2}$ & 0.00 & 0.05 & 0.10 & 0.50 & $\mu_{2} \geq 1$ or unknown \\
\hline Park and Ko [9] & 1.99 & 1.81 & 1.75 & 1.61 & 1.62 \\
Kim [14] & 2.52 & 2.17 & 2.02 & 2.59 & 1.60 \\
Theorem 4 & 3.03 & 2.82 & 2.78 & - & - \\
Corollary 8 & - & - & - & - & 1.64 \\
\hline
\end{tabular}

TABLE 3: Delay bounds $h$ with different $\mu_{2}$ and $\mu_{1}=-\mu_{2}$.

\begin{tabular}{lccccc}
\hline$\mu_{2}$ & 0.2 & 0.4 & 0.6 & 0.8 & $\mu_{2} \geq 1$ or unknown \\
\hline Li et al. [5] & 1.6063 & 1.4119 & 1.2425 & 1.1077 & - \\
Park et al. [29, Theorem 2] & 1.6492 & 1.5297 & 1.4489 & 1.3899 & - \\
Theorem 4 & 1.9464 & 1.7705 & 1.6646 & 1.5911 & - \\
Corollary 8 & - & - & - & - & 1.4924 \\
\hline
\end{tabular}

TABLE 4: Delay bounds $h$ with different $\mu_{2}$ and $\mu_{1}=-\mu_{2}$.

\begin{tabular}{lcccc}
\hline$\mu_{2}$ & 0.5 & 0.9 & $\mu_{2} \geq 1$ or unknown & Number of variables \\
\hline Li et al. [5] & 0.62 & 0.60 & - & 10 \\
He et al. [11] & 0.59 & 0.58 & - & 12 \\
He et al. [23] & 0.63 & 0.62 & - & 14 \\
Theorem 4 & 0.68 & 0.67 & 0.66 & 9 \\
Corollary 8 & - & - & 7 \\
\hline
\end{tabular}

Example 10. Consider the linear system (1) with the parameters

$$
A=\left[\begin{array}{cc}
0 & 1 \\
-1 & -1
\end{array}\right], \quad B=\left[\begin{array}{cc}
0 & 0 \\
0 & -1
\end{array}\right]
$$

With the conditions $0 \leq h(t) \leq h$ and $\mu_{1} \leq \dot{h}(t) \leq \mu_{2}<1$, our results obtained by Theorem 4 with the above systems are shown in Table 2. When $h(t)$ is not differentiable or $\dot{h}(t)$ is unknown, the corresponding results obtained by Corollary 8 are also included in Table 2. From Table 2, it can be seen that our results obtained both by Theorem 4 and by Corollary 8 improve the allowable maximum size of the delay for various $\mu_{2}$.

Example 11. Consider the linear system (1) with the parameters

$$
A=\left[\begin{array}{cc}
-0.9 & 0.2 \\
0.1 & -0.9
\end{array}\right], \quad B=\left[\begin{array}{cc}
-1.1 & -0.2 \\
-0.1 & -1.1
\end{array}\right]
$$

Our results obtained by Theorem 4 and by Corollary 8 are listed in Table 3. From Table 3, one can see that our results for Example 11 give larger upper bounds of time delay than the ones in $[5,29]$.
Example 12. Consider the linear system (1) with the parameters

$$
\begin{gathered}
A=\left[\begin{array}{cccc}
-1 & 0 & 1 & 0 \\
0 & -1 & 0 & 1 \\
-0.009 & 0.09 & -0.04 & 0.04 \\
0.09 & 0.09 & 0.04 & -0.06
\end{array}\right], \\
B=\left[\begin{array}{cccc}
1 & 0 & 0 & 0 \\
0 & 1 & 0 & 0 \\
-1.1789 & -1.3096 & -1.6629 & -7.3974 \\
0 & 0 & 0 & 0
\end{array}\right] .
\end{gathered}
$$

Table 4 lists the comparison results for $\mu_{2}$ and unknown $\mu_{2}$. It is clear that the results obtained in this paper are better than those in $[5,11,23]$. We can also see that the number of variables of our paper is less than that of others, so our methods can reduce the computational burden.

\section{Conclusion}

The problem of delay-dependent stability for linear system with time-varying delay is investigated in this paper. By using a novel combined convex technique and Wirtinger inequality to deal with the derivative of Lyapunov-Krasovskii functional, a less conservative delay-dependent stability criterion expressed in terms of LMIs has been presented. Four illustrative examples are given to demonstrate the reduced 
conservativeness of the proposed method and improvements over some existing ones.

\section{Conflict of Interests}

The authors declare that there is no conflict of interests regarding the publication of this paper.

\section{Acknowledgment}

This work is supported by the National Nature Science Foundation under Grant 61374154.

\section{References}

[1] Y. Ariba and F. Gouaisbaut, "An augmented model for robust stability analysis of time-varying delay systems," International Journal of Control, vol. 82, no. 9, pp. 1616-1626, 2009.

[2] P. Niamsup, K. Mukdasai, and V. N. Phat, "Improved exponential stability for time-varying systems with nonlinear delayed perturbations," Applied Mathematics and Computation, vol. 204, no. 1, pp. 490-495, 2008.

[3] W. Qian, S. Cong, Y. Sun, and S. Fei, "Novel robust stability criteria for uncertain systems with time-varying delay," Applied Mathematics and Computation, vol. 215, no. 2, pp. 866-872, 2009.

[4] S. Xu and J. Lam, "On equivalence and efficiency of certain stability criteria for time-delay systems," IEEE Trans on Automatic Control, vol. 52, no. 1, pp. 95-101, 2007.

[5] T. Li, L. Guo, and L. Wu, "Simplified approach to the asymptotical stability of linear systems with interval time-varying delay," IET Control Theory \& Applications, vol. 3, no. 2, pp. 252-260, 2009.

[6] P. Park, "A delay-dependent stability criterion for systems with uncertain time-invariant delays," IEEE Transactions on Automatic Control, vol. 44, no. 4, pp. 876-877, 1999.

[7] E. Fridman and U. Shaked, "An improved stabilization method for linear time-delay systems," IEEE Transactions on Automatic Control, vol. 47, no. 11, pp. 1931-1937, 2002.

[8] S. K. Nguang, P. Shi, and S. Ding, "Delay-dependent fault estimation for uncertain time-delay nonlinear systems: an LMI approach," International Journal of Robust and Nonlinear Control, vol. 16, no. 18, pp. 913-933, 2006.

[9] P. G. Park and J. W. Ko, "Stability and robust stability for systems with a time-varying delay," Automatica, vol. 43, no. 10, pp. 18551858, 2007.

[10] W. Qian, S. Cong, T. Li, and S. Fei, "Improved stability conditions for systems with interval time-varying delay," International Journal of Control, Automation and Systems, vol. 10, no. 6, pp. 1146-1152, 2012.

[11] Y. He, Q. Wang, C. Lin, and M. Wu, "Delay-range-dependent stability for systems with time-varying delay," Automatica, vol. 43, no. 2, pp. 371-376, 2007.

[12] E. Fridman and U. Shaked, "A descriptor system approach to $H_{\infty}$ control of linear time-delay systems," IEEE Transactions on Automatic Control, vol. 47, no. 2, pp. 253-270, 2002.

[13] R. Sipahi, S. Niculescu, C. T. Abdallah, W. Michiels, and K. $\mathrm{Gu}$, "Stability and stabilization of systems with time delay: limitations and opportunities," IEEE Control Systems Magazine, vol. 31, no. 1, pp. 38-65, 2011.
[14] J. Kim, "Note on stability of linear systems with time-varying delay," Automatica, vol. 47, no. 9, pp. 2118-2121, 2011.

[15] O. M. Kwon, S. M. Lee, J. H. Park, and E. J. Cha, "New approaches on stability criteria for neural networks with interval time-varying delays," Applied Mathematics and Computation, vol. 218, no. 19, pp. 9953-9964, 2012.

[16] X. Jiang and Q. L. Han, "New stability criteria for linear systems with interval time-varying delay," Automatica, vol. 44, no. 10, pp. 2680-2685, 2008.

[17] H. Shao, "New delay-dependent stability criteria for systems with interval delay," Automatica, vol. 45, no. 3, pp. 744-749, 2009.

[18] A. Seuret and F. Gouaisbaut, "Wirtinger-based integral inequality: application to time-delay systems," Automatica, vol. 49, no. 9, pp. 2860-2866, 2013.

[19] Z. G. Wu, J. H. Park, H. Su, and J. Chu, "New results on exponential passivity of neural networks with time-varying delays," Nonlinear Analysis: Real World Applications, vol. 13, no. 4, pp. 1593-1599, 2012.

[20] J. Sun, G. P. Liu, J. Chen, and D. Rees, "Improved delay-rangedependent stability criteria for linear systems with time-varying delays," Automatica, vol. 46, no. 2, pp. 466-470, 2010.

[21] K. Liu and E. Fridman, "Wirtinger's inequality and Lyapunovbased sampled-data stabilization," Automatica, vol. 48, no. 1, pp. 102-108, 2012.

[22] D. Yue, E. Tian, Y. Zhang, and C. Peng, "Delay-distributiondependent stability and stabilization of T-S fuzzy systems with probabilistic interval delay," IEEE Transactions on Systems, Man, and Cybernetics B: Cybernetics, vol. 39, no. 2, pp. 503-516, 2009.

[23] Y. He, Q. Wang, L. Xie, and C. Lin, "Further improvement of free-weighting matrices technique for systems with timevarying delay," IEEE Transactions on Automatic Control, vol. 52, no. 2, pp. 293-299, 2007.

[24] P. Park, J. W. Ko, and C. Jeong, "Reciprocally convex approach to stability of systems with time-varying delays," Automatica, vol. 47, no. 1, pp. 235-238, 2011.

[25] J. Jiao, "Delay-dependent stability criteria for singular systems with interval time-varying delay," Mathematical Problems in Engineering, vol. 2012, Article ID 570834, 16 pages, 2012.

[26] H. G. Zhang, Z. S. Wang, and D. R. Liu, "Global asymptotic stability and robust stability of a class of Cohen-Grossberg neural networks with mixed delays," IEEE Transactions on Circuits and Systems, I: Regular Papers, vol. 56, no. 3, pp. 616629, 2009.

[27] H. Zhang, Z. Wang, and D. Liu, "Global asymptotic stability of recurrent neural networks with multiple time-varying delays," IEEE Transactions on Neural Networks, vol. 19, no. 5, pp. 855873, 2008.

[28] W. Qian and H. R. Karimi, "New delay-dependent stability conditions for time-varying delay systems," Mathematical Problems in Engineering, vol. 2013, Article ID 360924, 6 pages, 2013.

[29] M. J. Park, O. M. Kwon, J. H. Park, and S. M. Lee, "A new augmented Lyapunov-Krasovskii functional approach for stability of linear systems with time-varying delays," Applied Mathematics and Computation, vol. 217, no. 17, pp. 7197-7209, 2011.

[30] Q. L. Han, "A discrete delay decomposition approach to stability of linear retarded and neutral systems," Automatica, vol. 45, no. 2, pp. 517-524, 2009.

[31] X. M. Zhang and Q. L. Han, "Stability of linear systems with interval time-varying delays arising from networked control 
systems," in Proceedings of the 36th Annual Conference of the IEEE Industrial Electronics Society (IECON '10), pp. 225-230, November 2010.

[32] T. Li, T. Wang, A. Song, and S. Fei, "Combined convex technique on delay-dependent stability for delayed neural networks," IEEE Transactions on Neural Networks and Learning Systems, vol. 24, no. 9, pp. 1459-1466, 2013.

[33] I. E. Köse, F. Jabbari, and W. E. Schmitendorf, "A direct characterization of L2-gain controllers for LPV systems," IEEE Transactions on Automatic Control, vol. 43, no. 9, pp. 1302-1307, 1998. 


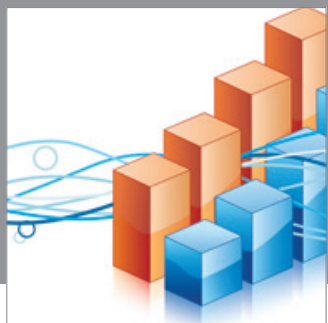

Advances in

Operations Research

mansans

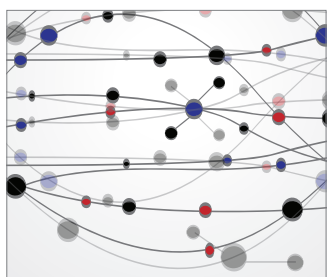

The Scientific World Journal
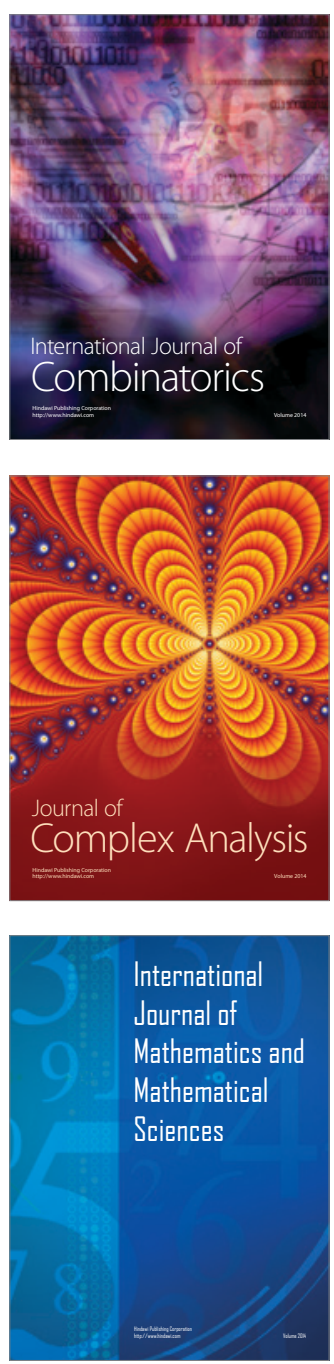
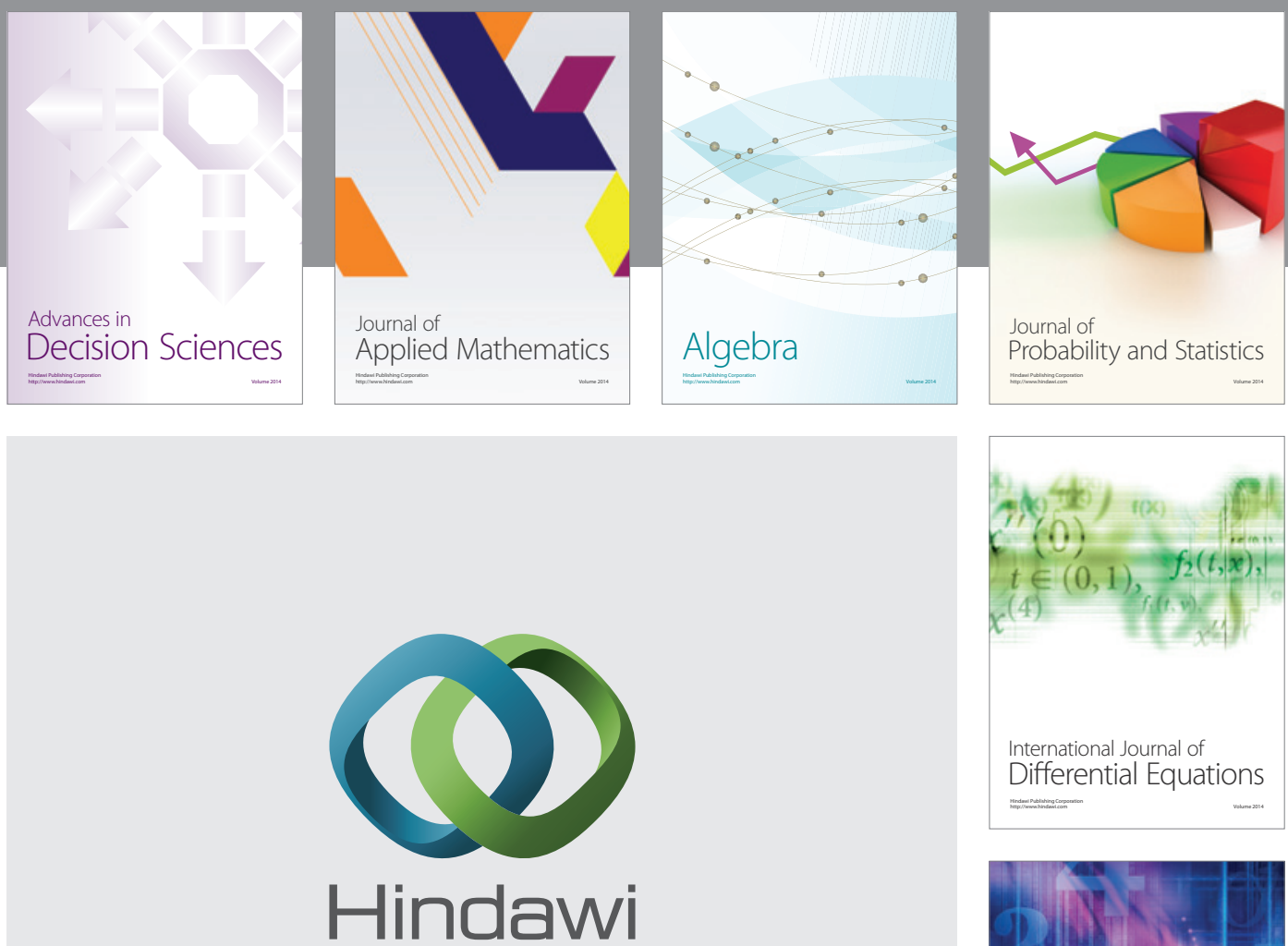

Submit your manuscripts at http://www.hindawi.com
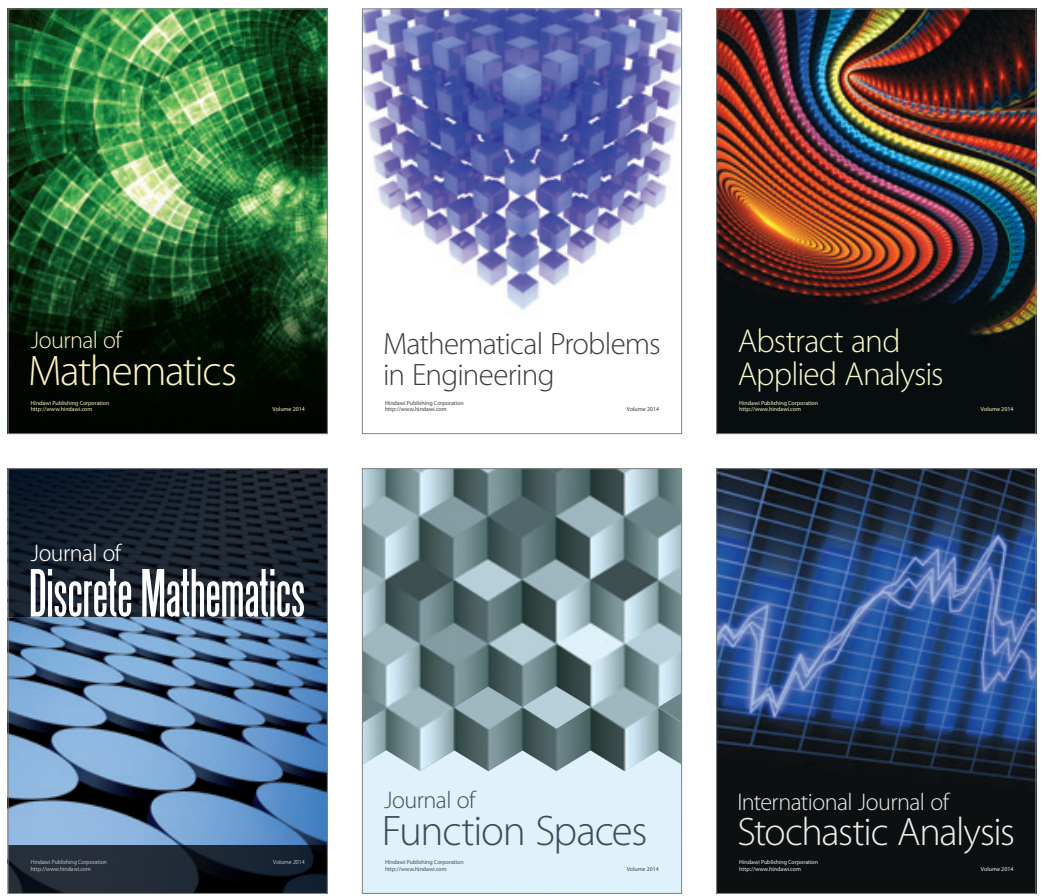

Journal of

Function Spaces

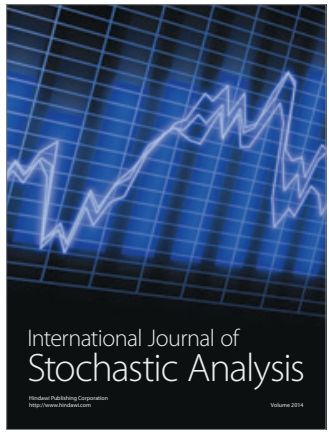

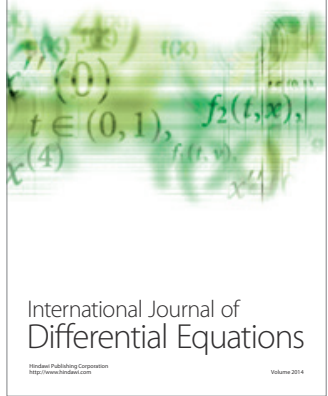
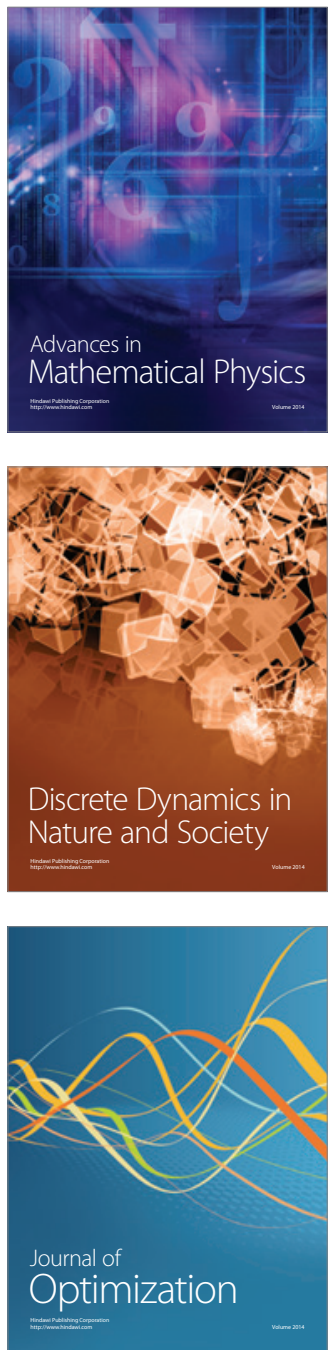